\title{
Measuring the mechanical behavior of individual nanoparticles inside a TEM
}

\author{
Zhiwei Shan*, Andrew M. Minor*, S.A. Syed Asif**, O.L. Warren**, \\ * National Center for Electron Microscopy, LBL, Berkeley, CA, 94720 \\ **Hysitron Incorporated, Minneapolis, MN
}

The past two decades have witnessed remarkable advances in the processing and characterization of materials with characteristic length scales in the nanometer range ${ }^{1,2}$. The physical properties of nanomaterials are strongly dependent on their size, microstructure and chemistry ${ }^{3}$. To fully utilize the advantages offered by the size specificity and selectivity of nanomaterials, it is essential to investigate the unique characteristics of individual nanostructures, such as nanowires or nanoparticles with well-understood microstructures. Scanning probe microscopy techniques (STM, AFM) have proven to be a powerful tool and a dominant approach to characterizing the properties of individual nanostructures while transmission electron microscopy (TEM) has been traditionally applied to separately characterizing the microstructure of nanomaterials. By incorporating the advantages of these two kinds of instruments, we have recently developed a novel and unique in situ TEM nano-manipulator, which for the first time enables us to manipulate and quantitatively measure the force vs. displacement during mechanical deformation while simultaneously monitoring the microstructural evolution of individual nanostructures.

In this work, we report the current progress in applying our unique in situ TEM nano-manipulator (displacement resolution of $<0.5 \mathrm{~nm}$ and load resolution of $<0.5 \mu \mathrm{N}$ ) for mechanical measurement of nanocrystalline gold particles. The principle of this technique is to compress single particles between a specially-designed silicon wedge and a Berkovich diamond tip. The deformation processes were recorded with a TV-rate video-intensified camera and the extracted image sequence was used to calibrate the contact area. Quite unexpectedly, the Au particles are observed to deform in a discrete manner under the displacement control mode and the load vs time plots are at odds with typical concepts of materials deformation. The physical mechanism that accounts for this observed phenomenon will be discussed. In addition, internal defects, sample geometry, or sample sliding, which may not be identified with SPM devices, are found to have important effects on the measured materials strength. These findings suggest that in situ quantitative TEM is an irreplaceable tool for the nanomechanical tests ${ }^{4}$.

\section{References}

1. Gleiter, H. Nanostructured materials: Basic concepts and microstructure. Acta Materialia 48, $1-29$ (2000).

2. Wang, Z. L. Zinc oxide nanostructures: growth, properties and applications. J. Phys.: Condens. Matter 16 . R829-R858 (2004).

3. Wang, Z. L. Characterization of Nanophase Materials (Wiley-VCH, New York, 1999).

4. The authors acknowledge that the research was supported in part by a U.S. Department of Energy SBIR grant (DE-FG02-04ER83979) awarded to Hysitron, Inc., which does not constitute an endorsement by DOE of the views expressed in the article. This work also was supported by the Director, Office of Science, Office of Basic Energy Sciences, of the U.S. Department of Energy under Contract No. DE-AC02-05CH11231. 

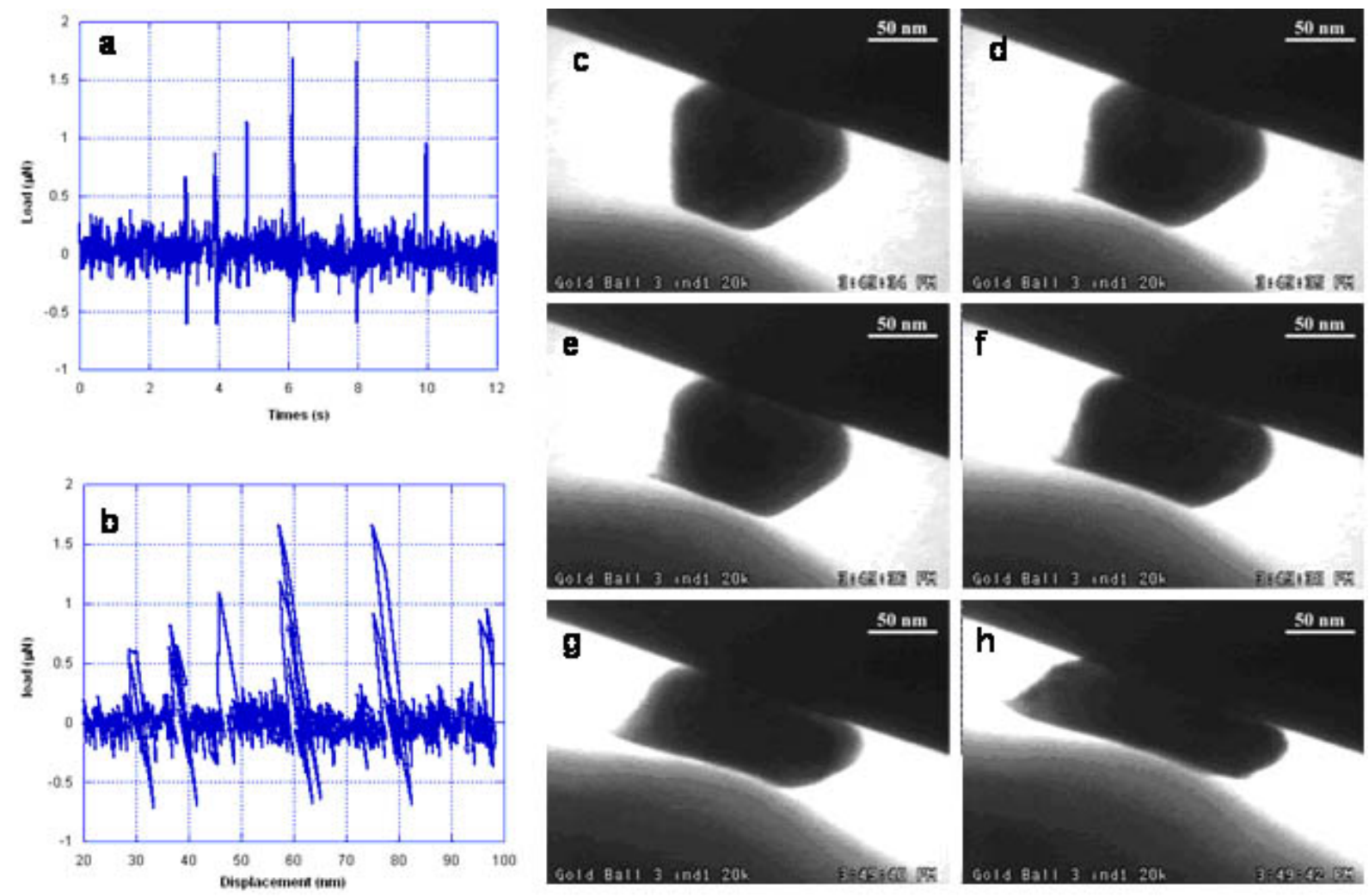

FIG. 1. Measuring the mechanical properties of nanocrystalline Au particles with an in situ TEM nano-manipulator. The load was performed under displacement control. a. Typical force vs time plot of the $\mathrm{Au}$ particle. Note that the deformation occurred in a discrete manner. $\mathrm{b}$. Typical load vs displacement curve. Figure 1 (c to f) are images extracted from video tape and corresponding the six load peaks shown in Fig. 1a and 1b, respectively. 